01

\title{
Самоподобие процесса десинхронизации в сети обобщенных осцилляторов Курамото
}

\author{
(C) А.А. Короновский ${ }^{1}$, М.К. Куровская ${ }^{1, \uparrow, ~ О . И . ~ М о с к а л е н к о ~}{ }^{1}$, \\ A.E. Храмов ${ }^{1,2}$ \\ ${ }^{1}$ Саратовский национальный исследовательский государственный \\ университет им. Н.Г. Чернышевского \\ ${ }^{2}$ Саратовский государственный технический университет \\ им. Гагарина Ю.А. \\ ฯ E-mail: mariakurovskaya@gmail.com
}

Поступило в Редакцию 5 июня 2017 г.

Рассматривается явление взрывной синхронизации в сети обобщенных осцилляторов Курамото. Показано, что данный процесс является следствием самоподобия, наблюдающегося при потере устойчивости синхронными кластерами разного размера. Проявление самоподобия может быть обнаружено благодаря исследованию процессов разрушения синхронного состояния сети. Продемонстрировано, что при резком разрушении синхронного режима система проходит через последовательность самоподобных конфигураций взаимодействующих осцилляторов.

DOI: 10.21883/PJTF.2017.19.45081.16901

Исследование динамики сложных сетей фазовых осцилляторов позволяет описать поведение большого числа реальных систем, таких как нейроны головного мозга, клетки сердечной мышцы, электросети и т.д. [1,2]. Явление синхронизации играет ключевую роль в коллективной динамике узловых элементов сети, при этом переходы между асинхронным и синхронным режимами имеют важное значение для понимания основных механизмов поведения взаимодействующих осцилляторов [3,4] и сложных сетей [5-7].

С термодинамической точки зрения переход от асинхронной динамики к синхронному режиму можно трактовать как фазовый переход. Различают два типа фазовых переходов: резкий переход к синхронному состоянию (так называемый фазовый переход первого рода) и 
непрерывный фазовый переход (фазовый переход второго рода) [8]. Обычно в сложных сетях наблюдается плавный переход от асинхронной динамики к синхронизации по мере увеличения параметра связи между узлами сети $[6,9]$. В то же время переход иного рода (фазовый переход первого рода), называемый взрывной синхронизацией, когда сеть не проходит плавно промежуточные частично синхронизованные состояния, а резко переходит скачком из асинхронного состояния в полностью синхронный режим, также наблюдается в динамике сложных сетей $[10,11]$. Известно, что возникновение взрывной синхронизации возможно в сетях с различным типом топологии связей. Фазовый переход первого рода к синхронной динамике имеет место в сетях с регулярной топологией связей, где каждый структурный элемент сети связан со всеми остальными узлами [12], в сетях со случайной топологией связей [13], а также в свободно масштабируемых сетях (scale-free networks) $[14,15]$, в том числе в свободно масштабируемых сетях, характеризующихся межэлементными связями с временно́й задержкой [16]. Взрывная синхронизация также наблюдается в сетях осцилляторов с адаптивной связью [17]. Для возникновения фазового перехода первого рода (взрывной синхронизации) должен быть выполнен ряд условий, различных для сетей с разной топологией связей, при этом как установление, так и разрушение синхронного режима в этих случаях характеризуются резким качественным изменением динамики элементов сети, а в ряде случаев наблюдается гистерезис $[8,14]$.

Важно отметить, что, несмотря на то что наиболее популярной моделью для изучения взрывной синхронизации являются сети осцилляторов Курамото [18,19], спонтанное, мгновенно возникающее изменение в динамике сети осцилляторов, приводящее к резкому разрушению/установлению синхронного режима, наблюдается также и для других типов осцилляторов, когда они являются структурными элементами сложных сетей, например для кусочно-линейных систем Ресслера [10] или обобщенных осцилляторов Курамото [20]. Иными словами, резкий переход от синхронного состояния осцилляторов сети со сложной топологией межэлементных связей к асинхронной динамике (и наоборот) представляет собой универсальное явление, возникающее при определенных условиях в сложных сетях с различными узловыми элементами и разными типами топологии связей.

В настоящей работе на примере сети обобщенных осцилляторов Курамото [20] показывается, что резкое изменение состояния сети 
со сложной топологией связей - следствие явления самоподобия, когда разрушение синхронного режима происходит последовательно через самоподобные конфигурации взаимодействующих осцилляторов, теряющие устойчивость при одном и том же значении управляющего параметра, характеризующего интенсивность межэлементных связей.

Результаты, представленные в работе, были получены для сети обобщенных осцилляторов Курамото [20], состоящей из $N=5 \cdot 10^{3}$ элементов, динамика которых описывается уравнениями

$$
\dot{\theta}_{i}=\omega_{i}+\frac{\lambda\left|\omega_{i}\right|}{N} \sum_{j=1}^{N} \sin \left(\theta_{j}-\theta_{i}\right), \quad i=1, \ldots, N,
$$

где $\theta_{i}$ и $\omega_{i}$ - мгновенная фаза и собственная частота $i$-го осциллятора, точка означает производную по времени, $\lambda$ - параметр связи. В рамках рассматриваемой модели все осцилляторы сети связаны друг с другом, собственные частоты осцилляторов $\omega_{i}$ были заданы эквидистантно в интервале $[-0.5,0.5]$, начальные фазы осцилляторов без ограничения общности были выбраны равными нулю. Поскольку все значения собственных частот модифицированных осцилляторов Курамото различны, синхронная динамика в сети возникает только при значении параметра связи выше граничного значения $\lambda_{c}$, при этом все осцилляторы сети находятся в синхронизме, а сама сеть в целом может рассматриваться как единый синхронный кластер размера $N$. При критическом значении параметра связи $\lambda_{c}$ (для рассматриваемой сети $\lambda_{c}=2.0$ ) синхронный кластер начинает разрушаться [20].

Когда с уменьшением величины связи $\lambda$ при $\lambda^{*}$ синхронный кластер размера $N$ теряет устойчивость, часть осцилляторов выходит из синхронизма, а оставшиеся в синхронном состоянии осцилляторы составляют кластер размера $K<N$. В том случае, если новый кластер оказывается устойчивым при этом же самом значении параметра $\lambda^{*}$, сеть плавно переходит в новое устойчивое состояние, для разрушения которого нужно снова уменьшать интенсивность связи, что соответствует фазовому переходу второго рода [8]. Соответственно для того чтобы наблюдался фазовый переход первого рода, при котором происходит резкое разрушение синхронного состояния всей сети и переход к несинхронной динамике осцилляторов без существования крупных кластеров, все синхронные структуры различных размеров $K$, такие, что $K<N$, должны становиться неустойчивыми при одном и

Письма в ЖТФ, 2017, том 43, вып. 19 


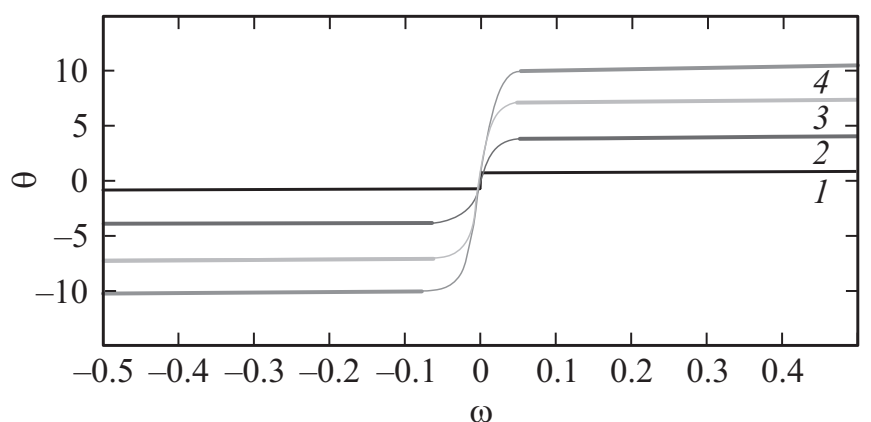

Зависимости фаз обобщенных осцилляторов Курамото сети (1) от их частот, полученные при значении $\lambda=1.99<\lambda_{c}$ в различные моменты времени: $1-$ $t_{1}=201,2-t_{2}=318,3-t_{3}=447,4-t_{4}=492$. Горизонтальные участки, показанные жирной линией, соответствуют осцилляторам, образующим в данный момент времени синхронный кластер, тонкая линия - элементам, демонстрирующим асинхронную динамику.

том же значении интенсивности связи $\lambda_{c}$. Иными словами, когда при $\lambda=\lambda_{c}$ начинается процесс разрушения синхронного состояния сети, часть осцилляторов начинает эволюционировать асинхронно и, как следствие, первоначальная когерентная структура размера $N$ заменяется на когерентную структуру меньшего размера (состоящую из $K(t)$ синхронных осцилляторов, $K(0)=N$ ), которая в свою очередь также неустойчива и заменяется на когерентную структуру $K(\tau)<K(t)$, $t<\tau$, и т.д. Поскольку синхронные кластеры всех размеров $K \leq N$ теряют устойчивость при одном и том же значении интенсивности связи, они должны обладать свойствами самоподобия. Таким образом, когда имеет место „взрывной“ переход от синхронизации к асинхронной динамике, когерентный кластер синхронных осцилляторов последовательно проходит через самоподобные конфигурации уменьшающегося размера $K(t)$, при этом все они становятся неустойчивыми при одном и том же значении параметра связи $\lambda=\lambda_{c}$.

Выявить самоподобный механизм, реализующийся при фазовом переходе первого рода в сети обобщенных осцилляторов Курамото, можно с помощью временно́го анализа процесса разрушения синхронного кластера при значении параметра связи чуть меньше критического. На рисунке представлены зависимости фаз $\theta_{i}$ от частот $\omega_{i}$ для эле-

Письма в ЖТФ, 2017, том 43, вып. 19 
ментов исследуемой сети (1), полученные в разные моменты времени $t_{1}<t_{2}<t_{3}<t_{4}$ при значении $\lambda=1.99$ Горизонтальные участки соответствуют осцилляторам, образующим синхронный кластер в каждый из рассмотренных моментов времени, точки на наклонных участках образованы осцилляторами, вышедшими из синхронизма. Как можно видеть, с течением времени размер синхронного кластера уменьшается, соответственно растет число асинхронных осцилляторов, но при этом остающиеся синхронными осцилляторы формируют структуры, являющиеся самоподобными по отношению друг к другу.

Таким образом, при фазовом переходе первого рода сеть обобщенных осцилляторов Курамото проходит через последовательность самоподобных конфигураций взаимодействующих осцилляторов, обусловливающих резкое разрушение синхронного режима. Важно отметить, что разрушение начинается после прохождения некоего порога и идет не хаотично, а через последовательность сокращающихся упорядоченных структур.

Работа выполнена при поддержке Российского научного фонда (проект № 14-12-00224). Авторы выражают благодарность рецензенту за конструктивные предложения.

\section{Список литературы}

[1] Boccaletti S., Latora V., Moreno V., Chavez M., Hwang D.-U. // Phys. Rep. 2006. V. 424. P. 175.

[2] Колоскова А.Д., Москаленко О.И. // Письма в ЖТФ. 2017. Т. 43. В. 10. C. 102.

[3] Rosenblum M.G., Pikovsky A.S., Kurths J. // Phys. Rev. Lett. 1997. V. 78. P. 4193.

[4] Hramov A.E., Koronovskii A.A., Kurovskaya M.K. // Phys. Rev. E. 2007. V. 75. P. 36205.

[5] Arenas A., Diaz-Guilera A., Pérez-Vicente C.J. // Phys. Rev. Lett. 2006. V. 96. P. 114102.

[6] Arenas A., Diaz-Guilera A., Kurths J., Moreno Y., Zhou C. // Phys. Rep. 2008. V. 469. P. 93.

[7] Богомолов С.А., Стрелкова Г.И., Schöll E., Анищенко В.С. // Письма в ЖТФ. 2016. V. 42. Р. 103.

[8] Boccaletti S., Almendral J.A., Guan S., Leyva I., Liu Z., Sendiña-Nadal I., Wang Z., Zou Y. // Phys. Rep. 2016. V. 660. P. 1.

[9] Moreno Y., Pacheco A.F. // Europhys. Lett. 2004. V. 68. P. 603.

Письма в ЖТФ, 2017, том 43, вып. 19 
[10] Leyva I., Sevilla-Escoboza R., Buldu J.M., Sendina-Nadal I., Gomez-Gardenes J., Arenas A., Moreno Y., Gomez S., Jaimes-Reategui R., Boccaletti S. // Phys. Rev. Lett. 2012. V. 108. P. 168702.

[11] Leyva I., Navas A., Sendina-Nadal I., Almendral J.A., Buldu J.M., Zanin M., Papo D., Boccaletti S. // Sci. Rep. 2013. V. 3. P. 1281.

[12] Pazó D. // Phys. Rev. E. 2005. V. 72. P. 46211.

[13] Zhu L., Tian L., Shi D. // Phys. Rev. E. 2013. V. 88. P. 42921.

[14] Gómez-Gardeñes J., Gomez S., Arenas A., Moreno Y. // Phys. Rev. Lett. 2011. V. 106. P. 128701.

[15] Peron T.K.D., Rodrigues F.A. // Phys. Rev. E. 2012. V. 86. P. 56108.

[16] Peron T.K.D., Rodrigues F.A. // Phys. Rev. E. 2012. V. 86. P. 16102.

[17] Danziger M.M., Moskalenko O.I., Kurkin S.A., Zhang X., Havlin S., Boccaletti S. // Chaos. 2016. V. 26. P. 65307.

[18] Kuramoto Y. // Lect. Notes Phys. 1975. V. 30. P. 420.

[19] Acebrón J.A., Bonilla L.L., Pérez-Vicente C.J., Ritort F., Spigler R. // Rev. Mod. Phys. 2005. V. 77. P. 137.

[20] Hu X., Boccaletti S., Huang W., Zhang X., Liu Z., Guan S., Lai C.-H. // Sci. Rep. 2014. V. 4. P. 7262. 